\title{
Atención al paciente con diabetes: algo más que insulinas
}

\author{
A. Carcavilla Urquí \\ Pediatra. Servicio de Pediatría. Hospital Virgen de la Salud. \\ Toledo. España.
}

Rev Pediatr Aten Primaria. 2009; I I Supl 16:s217-s238

Atilano Carcavilla Urquí, acarcavilla@gmail.com

\section{Resumen}

El manejo de la diabetes tipo 1 en la infancia y adolescencia ha evolucionado en los últimos años, como consecuencia de la intensificación del tratamiento insulínico y la aceptación de nuevos objetivos en el tratamiento. La nutrición y el ejercicio se consideran elementos básicos para el control glucémico, y el autocontrol es la clave para una terapia exitosa. Para conseguir un manejo adecuado de la enfermedad es esencial una educación diabetológica apropiada, así como una motivación continua. El pediatra de Atención Primaria puede ayudar a los pacientes y sus familias en estos aspectos, y jugar un papel esencial en la detección precoz de la enfermedad. Asimismo, el personal de Atención Primaria debe estar preparado para hacer frente a situaciones comunes en la vida del pacientes con diabetes como las hipoglucemias y las infecciones, y prestar atención a los aspectos psicológicos en el cuidado de los pacientes y sus familias.

Palabras clave: Diabetes mellitus tipo 1, Nutrición, Educación diabetológica, Autocontrol, Hipoglucemia, Cetosis.

\section{Abstract}

Management of type 1 diabetes in infancy and childhood has evolved in the recent years, due to the intensification of treatment and the recognition of new targets in childhood diabetes' treatment. Dietary management and exercise remain critical components in blood glucose regulation, and monitoring of glycemic control is the key to a successful outcome. Self-management education and continuous motivation are essential to manage the disease. The Primary Care paediatrician provides a strong aid to support the patient and his family in these issues, and can play a major role in early detection. Furthermore, primary care professionals should be able to manage situations as hypoglycaemia and infections, and must pay attention to the psychological care of these patients and their families.

Key words: Type 1 diabetes mellitus, Nutritional management, Diabetes education, Self-monitoring, Hypoglycemia, Ketosis.

El autor declara no presentar conflictos de intereses en relación con la preparación y publicación de este artículo. 


\section{Introducción}

Se entiende por diabetes mellitus un grupo de enfermedades metabólicas caracterizadas por hiperglucemia crónica, resultado de defectos en la secreción y/o acción de la insulina ${ }^{1}$. Los criterios actuales de diabetes están basados en la medición de glucosa en sangre y la presencia o ausencia de síntomas².

Este artículo se refiere concretamente a la diabetes mellitus tipo 1 (DM1), caracterizada por la deficiencia absoluta de secreción de insulina y la tendencia a cetoacidosis, en relación con una patogénesis autoinmune. Para el resto de diabetes las siguientes consideraciones pueden ser de utilidad, pero algunas recomendaciones deben individualizarse y ampliarse.

En los últimos años, el manejo de la DM1 en la infancia y adolescencia ha cambiado considerablemente. Algunos de los factores implicados en este cambio podrían ser:

- La irrupción de las nuevas insulinas en la batería terapéutica y el desarrollo de la tecnología de los infusores subcutáneos de insulina. Variedades que permiten una administración más fisiológica de la insulina exógena y aportan mayor libertad de ingesta; y la realización de ejercicio, mejorando la calidad de vida de los pacientes.
- Existe mayor concienciación de que un control apropiado durante la infancia es tan necesario, o aún más, de lo que lo es en la etapa adulta. El Diabetes Control and Complications Trial aportó evidencias sólidas de que la intensificación del tratamiento insulínico reduce las complicaciones microvasculares y de que dicha intensificación requiere un control adecuado de la diabetes ${ }^{3}$.

- Ha habido un aumento en la incidencia de esta patología en la edad pediátrica, particularmente en menores de 5 años ${ }^{4}$.

Por todo lo anterior, resulta de especial interés revisar la evidencia disponible acerca de la DM1 en la infancia y adolescencia, y las recomendaciones actuales de las principales sociedades científicas, más allá de las referentes al tratamiento insulínico.

\section{Presentación y fases de la DM1}

Se caracteriza por cuatro fases:

\section{Diabetes preclínica}

Hace referencia a los meses o años que preceden a la presentación clínica. En esta fase ya se producen anticuerpos contra las células beta del páncreas, que pueden detectarse en suero. La conti- 
Tabla I. Criterios para el diagnóstico de las alteraciones del metabolismo hidrocarbonado²

\begin{tabular}{lccc}
\hline & $\begin{array}{c}\text { Diabetes } \\
\text { mellitus }\end{array}$ & $\begin{array}{c}\text { Alteración de la } \\
\text { glucosa en ayunas }\end{array}$ & $\begin{array}{c}\text { Alteración de la } \\
\text { tolerancia a la glucosa }\end{array}$ \\
\hline Glucemia en ayunas & $\geqslant 126 \mathrm{mg} / \mathrm{dl}^{*}$ & $100-125 \mathrm{mg} / \mathrm{dl}$ & - \\
\hline $\begin{array}{l}\text { Glucemia } 2 \text { horas tras una } \\
\text { sobrecarga oral de glucosa }\end{array}$ & $\geqslant 200 \mathrm{mg} / \mathrm{dl} l^{* *}$ & - & $140-199 \mathrm{mg} / \mathrm{dl}$ \\
\hline
\end{tabular}

* Se define ayunas como la ausencia de ingesta calórica durante al menos 8 horas.

**Para cumplir este criterio se debe realizar una prueba de sobrecarga oral de glucosa de acuerdo a los criterios de la OMS. El diagnóstico de diabetes mellitus requiere la detección de cualquiera de estas dos alteraciones en dos días diferentes, o la detección de una glucemia $\geqslant 200 \mathrm{mg} / \mathrm{dl}$ en cualquier momento del día en un paciente con síntomas.

nua destrucción de estas células conduce a una pérdida progresiva de la reserva secretora de insulina, para finalizar casi con una absoluta deficiencia.

En la tabla I se recogen los criterios de diabetes mellitus, alteración de la glucosa en ayunas y alteración de la tolerancia a la glucosa. Ante la presencia de alteraciones glucémicas en la infancia sin cumplir criterios de diabetes hay que pensar en:

- Una fase incipiente de DM1.

- Una diabetes mellitus tipo 2 (DM2) (que asocia sobrepeso y datos de insulinorresistencia, como la acantosis nigricans).

- Una diabetes monogénica (que asocia un fuerte componente familiar).

- Una hiperglucemia de estrés (en el contexto de estrés por infecciones, por ejemplo).

En la DM1 es excepcional tener que recurrir a una sobrecarga oral de glucosa para el diagnóstico. A pesar del aumento de la incidencia de la DM2 en los niños y adolescentes, la DM1 sigue siendo el tipo de diabetes más frecuente en la infancia.

Hasta la fecha no existen medidas que permitan retrasar o prevenir el inicio de la DM1 ${ }^{1}$ por lo que, en ausencia de síntomas, rara vez está justificado realizar estudios para detectar anticuerpos o identificar un HLA de riesgo.

\section{Debut diabético}

La forma de debut puede variar desde una presentación no urgente, hasta la deshidratación severa y el shock. La clínica cardinal consiste en la aparición progresiva de poliuria, polidipsia, polifagia y pérdida de peso, y en el caso de que se desarrolle una cetoacidosis, el paciente sufre dolor abdominal, vómitos, deshidratación, polipnea y puede evolucionar hasta el shock y el coma. Sin embargo, a veces el debut adopta formas atípicas que debemos estar pre- 
parados para identificar. Una tira reactiva de orina es un método sencillo, sensible y no invasivo para excluir una diabetes de presentación menos típica. En caso de presentar glucosuria (resultado siempre patológico) y cetonuria (que en presencia de glucosuria, traduce déficit de insulina), una glucemia capilar superior a $200 \mathrm{mg} / \mathrm{dl}$ confirma el diagnóstico'.

Formas de presentación no urgente de diabetes':

- Vómitos.

- Enuresis en un niño que ya tenía control de esfínteres.

- Candidiasis vaginal, sobre todo en chicas prepúberes.

- Pérdida de peso o fallo de medro.

- Irritabilidad o empeoramiento del rendimiento escolar.

- Infecciones cutáneas recurrentes.

Situaciones que pueden resultar en un diagnóstico tardío de cetoacidosis diabética (CAD):

- Niños muy pequeños, porque la $C A D$ se puede desarrollar rápidamente y es un diagnóstico que no se suele considerar a esa edad.

- La polipnea en CAD se puede confundir con asma o neumonía.

- El dolor abdominal, típico de la cetosis, puede confundirse con un abdomen agudo.
- La poliuria y enuresis se pueden interpretar como parte de una infección urinaria.

- Los vómitos podrían considerarse en relación con una gastroenteritis o sepsis.

Si se realiza un diagnóstico de diabetes en un niño con síntomas, se debe remitir urgentemente a un centro hospitalario para su estudio y tratamiento.

\section{Remisión parcial o luna de miel}

En aproximadamente un $80 \%$ de pacientes, los requerimientos de insulina disminuyen transitoriamente tras el inicio de la insulinoterapia. Una definición utilizada en la actualidad es cuando el paciente necesita menos de 0,5 unidades de insulina por kilo (las necesidades de insulina normalmente oscilan entre 0,5 $\mathrm{U} / \mathrm{kg}$ en niños prepúberes y hasta 1,5 $\mathrm{U} / \mathrm{kg}$ en adolescentes) y la $\mathrm{HbA} 1 \mathrm{c}$ es menor de $7 \%{ }^{5}$. Se suele presentar a los días-semanas de iniciar la insulinoterapia, y habitualmente dura meses (la duración media es de aproximadamente 10-12 meses y puede durar hasta 2 años).

En esta etapa es fundamental:

- Informar del carácter transitorio de esta fase, que en ningún caso evoluciona a la remisión total ${ }^{6}$.

- Estar atento a la disminución de las necesidades de insulina, que obliga 
con frecuencia a reducir las unidades del tratamiento y en ocasiones hasta suspender su administración en algún momento del día.

\section{Fase crónica}

Se trata de un proceso gradual de agotamiento de las células beta, que puede verse acelerado por un proceso infeccioso. En ocasiones, los pacientes con diabetes y sus familias intentan retrasar el reinicio de la insulinoterapia, por ejemplo evitando tomar hidratos de carbono en algún momento del día. Como se verá en el apartado de nutrición, esta actitud es claramente desaconsejable y se debe animar al paciente a usar insulina cuando la necesite, al seguir una dieta equilibrada con un $50-55 \%$ de aporte calórico en forma de hidratos de carbono.

No hay que olvidar que la DM1 es una enfermedad autoinmune, y como tal asocia otros procesos autoinmunes. Desde la consulta de diabetes se procede al cribado sistemático y periódico de otros procesos, como la enfermedad celíaca ${ }^{7}$ la tiroiditis autoinmune ${ }^{8}$.

\section{Educación diabetológica}

Se trata de la clave para conseguir un buen control metabólico. En la era del tratamiento intensivo de la diabetes, la educación diabetológica es fundamental para asegurar buenos resultados. Tanto porque es necesario transmitir una serie de conocimientos para el adecuado autocontrol, como porque es necesario un apoyo y una motivación constantes. Y porque además, ha demostrado tener un impacto positivo no solo en el control glucémico, sino sobre todo en aspectos psicosociales ${ }^{10}$ de los pacientes y sus familias. Existen múltiples guías publicadas ${ }^{11,12}$ que sirven de ayuda a la hora de elaborar un programa educativo.

Algunos principios básicos de la educación diabetológica en la infancia y la adolescencia son:

- Todos los familiares y encargados de cuidar del niño deben ser incluidos en el proceso educativo y tener acceso libre al mismo.

- La educación diabetológica debe estar estructurada en un programa que se adapte a las necesidades y peculiaridades del paciente y su entorno. En este sentido, el currículo del programa variará considerablemente según se trate de un lactante, un niño o un adolescente. Igualmente, deberá adaptarse en la medida de lo posible a las peculiaridades culturales del paciente y su entorno. 
- Los profesionales encargados de impartir la educación diabetológica deben poseer entrenamiento en habilidades educativas y pedagógicas, para garantizar tanto la transmisión de conocimientos como el trabajo de actitudes.

- La educación diabetológica debe ser un proceso continuado y repetido a lo largo del tiempo para resultar efectivo.

\section{Nutrición}

El manejo nutricional es uno de los elementos clave para asegurar un buen control metabólico. Dado que la evidencia disponible en la edad pediátrica es escasa y débil, a la hora de proporcionar consejos apropiados para el manejo nutricional se recomienda sensibilidad frente a las necesidades individuales y pragmatismo más que dogmatismo.

\section{Objetivos/puntos básicos}

- La dieta debe proporcionar la energía y los nutrientes necesarios para un crecimiento y desarrollo óptimos, manteniendo un índice de masa corporal apropiado.

- Debe evitar la aparición de hipoglucemias e hiperglucemias. La insulina debe adaptarse al apetito del niño y al patrón de alimentación. En gene- ral, hay que evitar obligar a comer o negar una comida para controlar los niveles de glucemia.

- Organizar la dieta en tres comidas principales al día, más los tentempiés, permite aportar los nutrientes necesarios, controlar el peso, evitar el picoteo y los excesos, así como facilitar un horario para monitorizar la glucemia. Además de las comidas principales, se suele facilitar la ingesta de media mañana y la merienda, $y$, en general, es aconsejable tomar algo antes de acostarse. La cantidad y tipo de hidratos de carbono a tomar en estos tentempiés debe ser discutida con el equipo diabetológico.

- Se debe respetar las peculiaridades culturales, étnicas y familiares, así como las necesidades psicosociales del paciente.

- Toda la familia debe estar involucrada a la hora de hacer cambios en la dieta, orientados a conseguir una forma saludable de alimentarse.

- No se debe perder de vista el objetivo de mantener y preservar la calidad de vida del paciente y su entorno. No se puede hablar de alimentos prohibidos en diabetes. Alguno de ellos se debe consumir excepcionalmente, como las bebidas azucara- 
das, teniéndolo en cuenta a la hora de calcular las unidades de insulina $y$, a menudo, en el contexto de ingestas ricas en grasa y proteínas para enlentecer el vaciamiento gástrico. Una alternativa razonable para estas bebidas es recomendar la ingesta de refrescos "light". Sin embargo, la negación total y sistemática de este tipo de alimentos puede tener consecuencias psicológicas negativas $^{13}$.

- Disminuir el consumo de productos manufacturados y animar a aprender a cocinar productos frescos.

- Al igual que en la población general, no se recomienda el consumo de refrescos con gas. Hay que aconsejar tomar fundamentalmente agua.

\section{Aporte energético}

Habitualmente se calculan las necesidades diarias de aporte calórico en función de la edad: de base un aporte diario de $1.000 \mathrm{kcal}$, y por cada año de edad se suman $100 \mathrm{kcal}$. A partir de los 10 años las niñas habitualmente siguen la dieta de $2.000 \mathrm{kcal}$, que en los varones pueden ser de 2.000-2.500, e incluso 3.000 si hacen deporte. En todo caso, las necesidades energéticas de cada paciente dependen de muchas más variables que la edad. Por lo tanto, ese cálculo se debe utilizar como una estimación inicial que habrá que adaptar a las características del paciente, siempre y cuando sea el adecuado para garantizar un crecimiento óptimo.

La distribución de nutrientes es prácticamente superponible a la recomendable en cualquier niño:

- Hidratos de carbono: 50-55\%.

- Grasas: 30-35\%.

- Menos del 10\% grasas saturadas y ácidos grasos "trans".

- Menos del 10\% grasas poliinsaturadas.

- Menos del 10\% grasas monoinsaturadas (hasta un 20\% del total del aporte energético).

- Proteínas: 10-15\%.

- Fibra: 3 g/250 kcal de aporte energético diario (entre 15 y $30 \mathrm{~g} / \mathrm{día}$ ).

Se recomiendan alimentos saludables como pan integral, cereales, legumbres, frutas, verduras y lácteos desnatados o semidesnatados.

Consumo moderado de sacarosa (hasta un $10 \%$ del total).

Las necesidades de minerales y vitaminas son las mismas que las de cualquier otro niño. Por ello se recomienda el consumo diario de cinco raciones de fruta y verduras ${ }^{12}$. No se recomienda el uso de suplementos vitamínicos, salvo que se evidencie un déficit específico. 


\section{Herramientas y métodos educativos}

No existe evidencia sólida que permita escoger un método educativo frente a otro. Son premisas uniformemente aceptadas:

- Evitar ser excesivamente dogmático o intentar imponer un método que resulte demasiado difícil para el paciente y su entorno.

- El control metabólico permite confirmar el éxito del método empleado.

- La mayoría de las estrategias educativas parten de la base de que la cantidad y el tipo de carbohidratos presentes en cada comida son los principales determinantes de la respuesta postprandial.

\section{Sistema de raciones}

La cantidad de gramos de hidratos de carbono presentes en cada ingesta se calcula de forma que cada 10 gramos constituya una ración. De este modo se calcula el número de raciones diarias necesarias para cada paciente con diabetes y su distribución a lo largo del día, permitiendo el intercambio entre distintos alimentos siempre que mantenga el número total de raciones.

Existen manuales ${ }^{14}$ que informan del cálculo de raciones de hidratos de carbono presentes en cada alimento, de forma que, por ejemplo, $20 \mathrm{~g}$ de pan blanco,
$200 \mathrm{ml}$ de leche, y $100 \mathrm{ml}$ de zumo natural, equivalen todos ellos a una ración (10 g) de hidratos de carbono.

\section{Índice glucémico}

Mide la respuesta postprandial de la glucemia en función del tipo de alimento. En general, el consumo de alimentos con bajo índice glucémico permite reducir la glucemia postprandial ${ }^{15}$. Algunos ejemplos de alimentos con bajo índice glucémico son el pan integral, la pasta y las frutas ${ }^{14}$.

\section{Cociente insulina/hidratos de carbono}

Inicialmente, el paciente tiene una dieta con una cantidad de hidratos de carbono fija para cada momento del día, que el diabetólogo va modificando en función del hambre del niño y el control glucémico. Con el tiempo, la cantidad que come y cuándo come se puede ir variando, particularmente en los regímenes basal-bolus. En los usuarios de régimen de múltiples inyecciones al día o de infusores subcutáneos, existe la posibilidad de calcular un cociente que represente el número de unidades de insulina necesarias por cada ración que se ingiere, para cada horario de comida. De esa forma, si el paciente quiere tomar menos cantidad de carbohidratos, usando el cociente puede calcular cuántas unidades necesita 
para esa comida. Este sistema no ha demostrado sólidamente su eficacia para mejorar el control glucémico, pero en todos los estudios demuestra una mejora de la calidad de vida de los pacientes.

\section{Hipoglucemia}

La hipoglucemia resulta de un desajuste entre la dosis de insulina, los hidratos de carbono ingeridos, o el ejercicio reciente ${ }^{16}$. No existe un acuerdo uniforme en cuanto a qué valor concreto considerar hipoglucemia, si bien en la actualidad se habla de $70 \mathrm{mg} / \mathrm{dl}$ como el límite inferior a no sobrepasar ${ }^{16}$.

Las hipoglucemias habitualmente se acompañan de síntomas autonómicos (temblores, palidez, sudor frío, palpitaciones), y/o secundarios a disfunción neurológica (neuroglucopénicos, como la dificultad para concentrarse, la visión borrosa, los mareos, la pérdida de conciencia o las convulsiones). Asimismo, los niños con hipoglucemia pueden presentar alteraciones conductuales como irritabilidad, Ilanto inconsolable... Más inespecíficos son algunos síntomas que pueden presentarse coincidiendo con cualquier valor de glucosa en sangre, como el hambre, el dolor de cabeza, el cansancio o las náuseas.

Sin embargo, los síntomas de hipoglucemia no siempre son fáciles de recono- cer en niños pequeños, y el umbral a partir del cual los pacientes pueden notarlos es variable, en función de si antes ha habido una hiper- o hipoglucemia. De tal forma que tras un episodio de hipoglucemia los síntomas pueden ver reducida su intensidad durante las siguientes 24 horas, con lo que es más fácil que las hipoglucemias pasen desapercibidas. De la misma manera, el paciente puede tener síntomas de hipoglucemia cuando la glucemia está bajando, a pesar de que se encuentre en un rango normal en ese momento.

Se considera hipoglucemia grave cuando el paciente no es capaz de ingerir por sí mismo los alimentos necesarios para resolverla. En estos casos se debe administrar glucagón intramuscular. La dosis es $10-30 \mathrm{mcg} / \mathrm{kg}$, pero en general se administra 0,5 mg en menores de 8 años o $25 \mathrm{~kg}$, y $1 \mathrm{mg}$ en mayores de 8 años o 25 kg. Cuando el nivel de conciencia se ha restablecido, se pasa a tratarlas como el resto de hipoglucemias.

El resto de hipoglucemias son leves o moderadas, y pueden a su vez ser sintomáticas o asintomáticas. Las hipoglucemias inadvertidas constituyen uno de los mayores factores de riesgo de hipoglucemia grave ${ }^{17}$.

En caso de hipoglucemia leve o moderada se debe: 
- Guardar reposo.

- Administrar hidratos de carbono de absorción rápida y hacer un control posterior a los 10-15 minutos para comprobar la respuesta.

- Si la hipoglucemia no se ha resuelto, hay que administrar más hidratos de absorción rápida hasta superarla.

- Una vez resuelta la hipoglucemia, ingerir hidratos de carbono de absorción lenta o tomar la siguiente comida, para prevenir la recurrencia de hipoglucemia.

La hipoglucemia ante todo debe ser prevenida. Existe evidencia de que las hipoglucemias frecuentes asocian deterioro cognitivo en niños con $\mathrm{DM} 1^{18}$, particularmente en los menores de 6 años ${ }^{19}$.

\section{Recomendaciones para la prevención} y el manejo de las hipoglucemias ${ }^{16}$

- El objetivo del tratamiento debe ser mantener la glucemia por encima de $70 \mathrm{mg} / \mathrm{dl}$, mientras se persigue el mejor control glucémico posible.

- Los pacientes, sus padres, profesores y otros cuidadores deben recibir instrucción acerca del reconocimiento y manejo de la hipoglucemia.

- Todos los pacientes deben llegar consigo un sistema de medición de glucemia en todo momento, así como hidratos de carbono de fácil absorción.

- Ante la presencia de síntomas, es prioritario administrar el tratamiento, aunque en ese momento no haya posibilidad de determinar la glucemia.

- Los familiares y cuidadores deben tener disponible glucagón, y saber cómo usarlo, sobre todo si el paciente tiene alto riesgo de hipoglucemia grave.

- Es recomendable que los niños y adolescentes lleven algún tipo de identificación que indique que tienen diabetes.

\section{Autocontrol}

Existen varias herramientas disponibles para asegurar un adecuado autocontrol, cada una juega un papel diferente, y son la clave para un adecuado control metabólico. Entre ellas destaca la HbA1c, la glucemia capilar y la determinación de cuerpos cetónicos en sangre y orina.

El Diabetes Control and Complications Trial ${ }^{3,20}$ proporcionó sólidas evidencias de que el control metabólico óptimo se asocia a complicaciones menos frecuentes $y$ más tardías. El parámetro que más evidencias ha ofrecido en ese sentido es la $\mathrm{HbA1c}$ : a menor $\mathrm{HbA1c}$, menos compli- 
caciones. Sin embargo, la HbA1c es una media de las glucemias que se han tenido en los últimos 3 meses, y como tal se ve afectada por los valores altos, tanto como por los bajos, de forma que una $\mathrm{HbA1c}$ puede corresponder a un control metabólico óptimo, pero también puede reflejar un exceso de hipoglucemias. En la actualidad, la opinión de los expertos coincide en que lo idóneo es conseguir una $\mathrm{HbA} 1 \mathrm{c}$ lo más cercana posible a la normalidad, evitando hipoglucemias y períodos prolongados de hiperglucemia.

En los últimos años, otro concepto se ha ido abriendo camino en el control de la diabetes, se trata de la variabilidad glucémica. Con la misma HbA1c, los pacientes con tratamiento intensivo tenían menos probabilidad de desarrollar retinopatía diabética que aquellos con tratamiento convencional ${ }^{21}$. $Y$ posteriormente, aunque de forma más errática, se han ido acumulando indicios de que una variabilidad glucémica mayor contribuye a una mayor incidencia de complicaciones ${ }^{22}$. Finalmente, numerosos estudios han incidido en la importancia de monitorizar con frecuencia la glucemia capilar y administrar la insulina correspondiente, teniendo en cuenta la ingesta de hidratos de carbono y el ejercicio realizado ${ }^{23}$. De tal forma que el pilar fundamental para un control metabólico óptimo y para retrasar e incluso evitar las complicaciones vasculares de la DM1, reside en la monitorización frecuente de la glucemia capilar para la toma de decisiones en cada momento y en el control periódico de la $\mathrm{HbA} 1 \mathrm{c}$ como indicador a medio plazo del control metabólico. Para mantener las glucemias dentro de la normalidad previniendo hipo- e hiperglucemias, es útil diseñar unas glucemias objetivo para cada paciente, que serán diferentes antes y después de la ingesta. En general, una recomendación razonable es mantener la glucemia preprandial entre 80 y $140 \mathrm{mg} / \mathrm{dl}$, y la postprandial entre 100 y $180 \mathrm{mg} / \mathrm{dl}^{12}$, con lo que se previene la glucosuria, con su consiguiente pérdida de calorías por la orina y la hipoglucemia ${ }^{25}$. Con todo, estos objetivos se deben individualizar en cada paciente en función de factores como la edad $^{24}$, fase de la enfermedad, capacidad de cumplimiento terapéutico, frecuencia de monitorización...

Otro elemento importante en el autocontrol es la realización de tiras reactivas para cuerpos cetónicos en orina o en sangre. Cuando esté disponible, la determinación en sangre es más fiable que en orina, por varios motivos. La determinación de cuerpos cetónicos en sangre detecta el $\beta$-hidroxibutirato, mientras que en orina detecta el aceto- 
acetato, que está en menor concentración que el $\beta$-hidroxibutirato. Por otra parte, los cuerpos cetónicos en orina reflejan su producción en sangre entre 4 y 12 horas antes. La mayoría de los estudios han demostrado que la determinación de cuerpos cetónicos en sangre es más útil que en orina para evitar las visitas a Urgencias ${ }^{26}$, y que la determinación en orina no es de mucha ayuda para diagnosticar o descartar una cetoacidosis ${ }^{27}$. En cualquier caso, se debe determinar cuerpos cetónicos en los siguientes casos ${ }^{28}$ :

- Enfermedad aguda con fiebre y/o vómitos.

- Niveles de glucemia persistentemente elevados por encima de 250 $\mathrm{mg} / \mathrm{dl}$, pese a tratamiento insulínico corrector.

- Poliuria persistente con hiperglucemia o glucosuria.

- Dolor abdominal o polipnea.

Para que el autocontrol sirva en la mejora del control glucémico, es fundamental que los pacientes y sus familias lleven un registro diario ${ }^{12}$ de las glucemias obtenidas, el número y tipo de raciones consumidas en cada horario y la duración y tipo de ejercicio realizado. Esto permite realizar las correcciones oportunas en el tratamiento desde la consulta, o por parte de los mismos pacientes.
Cómo actuar cuando el paciente con diabetes tiene una enfermedad aguda

Las infecciones agudas, sobre todo si cursan con fiebre, tienden a elevar la glucemia, como resultado de la elevación de las hormonas de estrés en sangre, entre otras el cortisol, que tienen un efecto contrarregulador y estimulan la neoglucogénesis. Por este mismo motivo, no es infrecuente que las necesidades de insulina aumenten desde unos días antes de la aparición de los síntomas, e igualmente persistan más elevadas de lo habitual hasta días después de resuelto el proceso.

Las infecciones digestivas, como la gastroenteritis aguda, pueden provocar tendencia a la hipoglucemia resultado de los vómitos, la escasa ingesta, la peor absorción intestinal y el vaciamiento gástrico enlentecido propios de estos procesos.

En estos casos, se deben seguir una serie de recomendaciones generale ${ }^{29}$.

- Nunca interrumpir la administración de insulina. Un error muy común es suspender las dosis de insulina cuando el niño está enfermo, al entender que puede ser perjudicial por estar enfermo y comiendo menos, con lo que aumenta el riesgo de cetoacidosis.

- En todo caso, la dosis total de insulina puede aumentarse o disminuirse, prestando atención a si el 
niño tiene tendencia a la hipo- o la hiperglucemia.

- Monitorizar la glucemia capilar y los cuerpos cetónicos en orina o sangre más frecuentemente.

- Cuando un niño con diabetes vomita, debe considerarse un signo de déficit de insulina hasta que se demuestre lo contrario.

Un niño con diabetes con una infección intercurrente debe recibir tratamiento apropiado para la infección de que se trate. Si el paciente necesita tratamiento con antitérmicos o antibióticos, deben ser pautados y administrados. Algunos preparados comerciales de ibuprofeno y de antibiótico en comprimidos carecen de hidratos de carbono entre sus excipientes. Con todo, si contienen hidratos de carbono no contraindican su uso. Únicamente hay que contabilizar los gramos de hidratos de carbono presentes en cada dosis (con frecuencia inferior a $5 \mathrm{~g}$ ), que se pueden calcular con una ojeada al prospecto, $y$ tenerlo en cuenta al contabilizar las raciones. A menudo las cantidades son despreciables, o al menos se pueden administrar junto a las comidas teniéndolo en cuenta a la hora de calcular las raciones y la insulina de cada ingesta.

En los pacientes en fase de "luna de miel" pueden aumentar las necesidades de insulina hasta requerimientos pro- pios de la fase de déficit total de insulina (hasta $1 \mathrm{U} / \mathrm{kg} /$ día). Cuando la enfermedad remite pueden volver a las necesidades de insulina previas, aunque tampoco es infrecuente que este proceso intercurrente precipite el final de la fase de remisión parcial o "luna de miel".

\section{Cuando hay tendencia}

a la hipoglucemia...

- En procesos con tendencia a la hipoglucemia, es frecuente que haya que disminuir la insulina basal y tener cuidado con las dosis de insulina rápida antes de cada ingesta. Puede suponer disminuir la insulina total diaria de un 20 a un $50 \%$.

- En caso de hipoglucemia, hay que tener cuidado de administrar el tratamiento correctamente, y no dar alimentos con grasas u otros productos que enlentezcan el vaciamiento gástrico hasta que no se haya resuelto la hipoglucemia (ver apartado de hipoglucemia).

- Cuando las hipoglucemias son frecuentes hay que aumentar el número de determinaciones de glucemia capilar con especial cuidado de no pasar por alto hipoglucemias inadvertidas, más frecuentes cuando se tiende a tener glucemias bajas. 
- En niños pequeños, y cuando estén inapetentes, es mejor administrar la insulina ultrarrápida inmediatamente después de la ingesta ${ }^{30}$, cuando sabemos el número de raciones real que ha tomado el paciente. Este sistema puede cubrir peor el pico de hiperglucemia postprandial inicial, pero es más seguro cuando el niño puede decidir tomar la mitad de las raciones que habíamos calculado, después de poner la insulina.

- Ante la pérdida de apetito, se puede administrar comidas de digestión fácil, sueros de rehidratación y líquidos azucarados, con lo que se administran calorías y se previene la cetoacidosis. De esa forma, las raciones que el paciente no ingiere en las comidas principales se pueden intercambiar por aquellas suministradas en forma de sueros de rehidratación.

- Hay que prestar atención al estado de hidratación. Algunas reglas que pueden ser de ayuda son registrar el volumen de ingesta, la frecuencia con que orina y el peso.

- Si coinciden hipoglucemia con cetonemia, generalmente refleja un aporte insuficiente de hidratos de carbono, que habrá que aumentar.
- Por último, a algunos de estos niños, sobre todo si son pequeños, puede ser necesario administrarles fluidos intravenosos si los vómitos o la diarrea persisten.

\section{Cuando hay tendencia}

a la hiperglucemia...

- Como norma general, aumentar el número de determinaciones de glucemia capilar. En estos casos además, es de especial ayuda realizar determinaciones de cuerpos cetónicos en orina o en sangre con frecuencia.

- En caso de hiperglucemia, se trata atendiendo a la necesidad de aumentar las unidades a administrar.

- Si hay hiperglucemia con cuerpos cetónicos negativos o "trazas" $(0$, $+/-0+/++++)$, se administra un $5-10 \%$ de insulina extra en forma de insulina rápida o ultrarrápida, se puede repetir cada 2-4 horas, ajustando los aportes en función de la respuesta.

- Si hay hiperglucemia con cetonuria moderada-alta $(++,+++,++++/$ $++++)$, aumentar los aportes de insulina diaria un $10-20 \%$ en forma de insulina rápida o ultrarrápida, repetir cada 2 horas, según evolu- 
ción de la glucemia y los cuerpos cetónicos. A menudo los cuerpos cetónicos asociados a hiperglucemia no se negativizan si no se administran además soluciones azucaradas en pequeñas cantidades, a partir del momento en que la glucemia desciende por debajo de los $250 \mathrm{mg} / \mathrm{d}^{25}$.

- Si hay vómitos asociados a hiperglucemia y cetonuria cederán tras administrar insulina, ya que frecuentemente se deben a cetosis por déficit de insulina.

\section{Recomendaciones sobre cuándo} acudir o remitir al servicio

\section{de Urgencias ${ }^{31}$}

- El proceso subyacente/intercurrente no está claro.

- Existen signos de deshidratación.

- Los vómitos persisten pasadas 2 horas.

- La glucemia tiende a elevarse a pesar de dosis extra de insulina.

- No se puede mantener la glucemia por encima de $70 \mathrm{mg} / \mathrm{dl}$.

- Cetonuria elevada o persistente a pesar de haberse administrado el tratamiento correcto.

- El niño está exhausto, confuso, hiperventilando o tiene dolor abdominal grave.
- Niños pequeños (menores de 2-3 años).

- Los padres están muy cansados.

- Problemas para comunicarse con el paciente y/o los familiares, por dificultades con el lenguaje.

\section{Ejercicio}

El ejercicio físico posee virtudes positivas para la salud, como reducir el riesgo cardiovascular, contribuir al control del peso y proporcionar sensación de bienes$\operatorname{tar}^{31}$. Se recomienda a los pacientes con diabetes hacer ejercicio físico de forma regular, dado que disminuye el riesgo de desarrollar complicaciones macrovasculares $^{12}$. En los niños con diabetes, el ejercicio debe tenerse en cuenta a la hora de calcular los hidratos de carbono que va a tomar y la insulina que se va a administrar, porque aumenta la sensibilidad a la insulina, pero también aumenta la síntesis y liberación de hormonas contrarreguladoras, que tienden a disminuir la sensibilidad a la insulina. La mayoría de los estudios publicados han sido incapaces de demostrar una mejoría de la $\mathrm{HbA1c}$ con el ejercicio ${ }^{33}$, aunque se piensa que podría deberse a los excesos para prevenir y corregir hipoglucemias ${ }^{34}$.

El ejercicio físico diario reglado es más fácil de manejar y de incorporar a las rutinas habituales del tratamiento. Lo idó- 
neo es que sea diario, con un mismo horario e intensidad, y aeróbico. Sin embargo, con frecuencia los niños hacen ejercicio no reglado, y su intensidad varía mucho día a día, cuando no de hora en hora. Para controlar y de hecho aprovechar los cambios que el ejercicio produce en el organismo del niño con diabetes, se debe tener en cuenta algunas consideraciones referentes al control metabólico, la ingesta de carbohidratos y la administración de insulina, en función también del tipo de ejercicio.

\section{Control metabólico}

Es conveniente, sobre todo cuando el ejercicio no es el habitual, realizar controles de glucemia capilar antes y después del ejercicio, así como tenerlo en cuenta a la hora de realizar nuevas determinaciones en las 12-24 horas siguientes, que también se pueden ver influenciadas por el ejercicio. Asimismo, durante la realización de ejercicio puede ser necesario realizar controles adicionales si es muy prolongado o aparecen síntomas de hipoglucemia.

Posteriormente se debe registrar las glucemias, la ingesta de carbohidratos y el tipo y duración del ejercicio realizado, que servirá para conocer la respuesta de nuestro paciente a ese tipo de ejercicio, y planificar futuras modificaciones en función del ejercicio que vaya a realizar.
Cuando hay mal control y cuerpos cetónicos positivos, el ejercicio puede aumentar la glucemia y provocar o acelerar una cetoacidosis, por tanto cuando la glucemia sea muy elevada (> $250 \mathrm{mg} / \mathrm{dl}$ ) hay que determinar cuerpos cetónicos en sangre u orina. De ser positivos, no debe realizar ejercicio, de ser negativos podrá realizar si en ese momento está bajo el efecto de alguna insulina previa ${ }^{25}$.

\section{Ingesta de carbohidratos}

- Para rellenar los depósitos de glucógeno hepático de un niño que va a realizar una actividad deportiva, es conveniente haber hecho una ingesta rica de hidratos de carbono, grasas y proteínas unas 3-4 horas antes del ejercicio.

- Cuando la glucemia anterior a la realización de ejercicio físico sea inferior a $120 \mathrm{mg} / \mathrm{dl}$, se aconseja tomar hidratos de carbono adicionales antes de la actividad ${ }^{12}$.

- Cuando el ejercicio se realiza durante el pico de acción de la insulina, puede tomar otros 5-10 g de hidratos de carbono (según el peso y la duración del ejercicio).

- Tras el ejercicio, en muchos casos es conveniente volver a rellenar los depósitos de glucógeno con la ingesta de hidratos de carbono. 
- En actividades anaeróbicas o muy competitivas, lo más frecuente es que la glucemia se eleve inmediatamente tras el ejercicio, en vez de disminuir. Sin embargo, horas después se deja notar el aumento de sensibilidad a la insulina, que hace recomendable la ingesta de carbohidratos al finalizar la actividad para prevenir hipoglucemias.

- Si durante el ejercicio el paciente se encuentra mal y tiene síntomas de hipo- o hiperglicemia, debe guardar reposo y tomar hidratos de carbono, incluso aunque no esté disponible un glucómetro.

\section{Administración de insulina}

- Tener cuidado de no administrar la insulina previa al ejercicio en los músculos que se van a ejercitar, porque aumenta la velocidad de absorción y su poder hipoglucemiante.

- Las hiperglucemias de estrés tras el ejercicio intenso no deben tratarse con insulina suplementaria ${ }^{17}, y$ hay que estar atento a la posible aparición de hipoglucemias tardías.

- Se recomienda disminuir las insulinas ultrarrápidas que se administran una hora antes del ejercicio, y las ultrarrápidas que se administran
3-4 horas antes ${ }^{35}$, pero si ha aumentado la ingesta de carbohidratos puede no ser necesario. Algunos niños que usan insulina ultrarrápida en la merienda, el realizar ejercicio por la tarde puede abolir la necesidad de insulina en ese horario; es más frecuente cuando queda reserva pancreática y cuando el ejercicio es de intensidad moderada y aeróbico. Con todo, las adaptaciones de las dosis de insulina deben individualizarse en cada paciente, en función de la respuesta del mismo al ejercicio.

- El aumento de la sensibilidad a la insulina se deja notar varias horas tras el ejercicio, y ha de tenerse en cuenta a la hora de administrar insulina en la siguiente ingesta (por ejemplo, tras realizar ejercicio por la tarde se necesita menos insulina con la cena). Aún más, algunos pacientes notan tendencia a la hipoglucemia por la noche los días de ejercicio, que obliga a un control más estrecho, e incluso en ocasiones a modificar la dosis de insulina basal que se administra esa noche; pueden utilizar una basal los días de ejercicio y otra los días de reposo.

- Cuando los niños se van de vacaciones o cambian de entorno, se 
justifica la necesidad de variaciones de la insulinoterapia, fundamentalmente como respuesta al cambio en la actividad diaria, aunque también intervienen otros factores.

- En los usuarios de infusores subcutáneos de insulina se puede programar una basal temporal más baja durante el ejercicio, e igualmente se debe considerar el ejercicio realizado a la hora de administrar los bolos posteriores. También se puede desconectar la bomba durante el ejercicio. Aunque para notar el efecto de disminución de la insulina basal debe hacerse con al menos 90 minutos de antelación al ejercicio, y, en general, no se recomienda desconectar la bomba más de 2 horas, existen evidencias de que desconectar la bomba durante el ejercicio se asocia con una menor probabilidad de hipoglucemias tardías ${ }^{36}$.

- Cuando se programa una actividad de un día entero, como una excursión, puede ser buena idea reducir los aportes de insulina basal en un 30-50\% el día de la actividad y el día siguiente.

\section{Aspectos psicológicos}

Existe evidencia de que los pacientes con DM1 tienen mayor riesgo de desa- rrollar problemas adaptativos los primeros años tras el diagnóstico ${ }^{37}$. El debut de la DM1 constituye una frustración frente a las expectativas de salud del paciente y sus allegados. En primer lugar, porque la enfermedad es algo inesperado, particularmente para un niño. $Y$, en segundo lugar, porque tras el alta, el paciente vuelve a casa y no está curado, su trastorno es crónico, y evitar que desarrolle complicaciones implica que él y las personas cercanas deben hacerse cargo de cosas que habitualmente hacen los sanitarios: controles de glucemia, administración subcutánea de medicamentos, toma de decisiones. Como tal frustración, el diagnóstico implica que la persona y sus familiares van a pasar por una serie de etapas de "elaboración de duelo" 38: negación, incomprensión, ira, culpa, indefensión... que pueden o no presentarse, entremezclarse y reaparecer. La única forma de negociar con la situación de una forma madura es reconocer cada una de estas etapas sin censurarlas, para progresivamente pasar a una fase de aceptación que permita desarrollar los cambios comportamentales necesarios para el autocontrol. Al inicio de la enfermedad, es posible que las fases de superación de la frustración no permitan a los pacientes y a sus cuidadores asimilar los conocimientos impartidos en la 
primera educación diabetológica, necesariamente realizada con inmediatez. Por eso es necesario insistir, reforzar, apoyar la educación inicial y motivar al paciente y a sus familiares constantemente. Está demostrado además que una actitud estimulante y motivadora en la educación puede conseguir un mejor cumplimiento terapéutico ${ }^{39}$.

Hay que prestar especial atención al entorno educativo de los niños con diabetes y promover su integración en el mismo. Para ello es fundamental garantizar una adecuada comunicación entre los padres y el centro, y potenciar todas las iniciativas disponibles para su mejor atención, como aumentar el personal sanitario en los centros y asegurar la disponibilidad de recursos de las asociaciones de diabéticos en el centro ${ }^{40}$.

Por último, se debe estar atento al eventual desarrollo de comorbilidad psiquiátrica en los pacientes y las personas de su entorno, ya que se ha demostrado que las personas con diabetes tienen mayor riesgo de desarrollar patología psiquiátrica que la población general, y en particular trastornos del comportamiento alimentario.

\section{Conclusiones}

El panorama de la DM1 ha cambiado considerablemente en los últimos años, y los avances en el diagnóstico y tratamiento permiten retrasar la aparición de complicaciones tardías con una mejoría considerable en la flexibilidad de horarios y actividades. Todas estas mejoras implican un adecuado autocontrol por parte del paciente y su entorno. Para conseguirlo es esencial una educación diabetológica de calidad que aporte información, facilite cambios comportamentales y motive al paciente. Desde la consulta de Atención Primaria se puede hacer mucho por reforzar la motivación del paciente y su entorno, absolutamente necesaria para un adecuado control metabólico. Asimismo, es un entorno privilegiado para facilitar la elaboración del duelo, y para la detección de trastornos asociados. Por último, es conveniente que el personal sanitario de Atención Primaria esté preparado para hacer frente a situaciones comunes en la vida del paciente con diabetes como las hipoglucemias, las infecciones, la planificación del ejercicio o los cambios de dieta. 


\section{Bibliografía}

1. ISPAD Clinical Practice Consensus Guidelines 2006-2007. Definition, epidemiology and classification. Pediatric Diabetes. 2006;7:343-51.

2. American Diabetes Association. Diagnosis and classification of diabetes mellitus. Diabetes $\mathrm{Ca}$ re. 2009;32:562-s67.

3. The diabetes control and complications trial research group. The effect of intensive treatment of diabetes on the development and progression of longterm complications in insulin-dependent diabetes mellitus. N Engl J Med. 1993;329:97786.

4. Eurodiab Ace Study Group. Variation and trends in incidence of childhood diabetes in Europe. Lancet. 2000;355:873-6.

5. Lombardo, $F$, Valenzise, $M$, Wasnieska $M$, et al. Two year prospective evaluation of the factors affecting honeymoon frequency and duration in children with insulin dependent diabetes mellitus: the key-role of age at diagnosis. Diabetes Nutr Metab Clin Exp. 2002;15:246-51.

6. The diabetes control and complications trial research group. Effect of intensive therapy on residual beta-cell function in patients with type 1 diabetes in the diabetes control and complications trial. A randomized, controlled trial. Ann Intern Med. 1998;128:517-23.

7. Nóvoa $Y$, López-Capapé M, Lara E, Alonso $M$, Camarero C, Barrio R. Impacto del diagnóstico de la enfermedad celíaca en el control metabólico de la diabetes tipo 1. An Pediatr (Barc). 2008;68 (1):13-7.

8. Roldán MB, Alonso M, Barrio R. Thyroid autoimmunity in children and adolescents with Type 1 diabetes mellitus. Diabetes Nutr Metab. 1999;12 (1):27-31.

9. Mensing C, Boucher J, Cypress M, et al. National standards for diabetes self-management education. Diabetes Care. 2005;28:s72-s79.
10. Winkley K, Ismail K, Landau S, et al. Psychological interventions to improve glycaemic control in patients with type 1 diabetes: systematic review and metaanalysis of randomised controlled trials. Br Med J. 2006;333:65-8.

11. Swift PGF. Diabetes education. Pediatric Diabetes. 2007;8:103-9.

12. National Institute for Clinical Excellence UK (NICE). Type 1 diabetes: diagnosis and management of type 1 diabetes in children, young people and adults [en línea]. 2004. Disponible en http://www.nice.org.uk/pdf/CG015NICEguide line.pdf

13. Aslander-van Vliet E, Smart C, Waldron S. Nutritional management in childhood and adolescent diabetes. Pediatric Diabetes. 2007;8:32339.

14. Luis F, Martínez-Contreras LF, Matas D, Ceñal MJ, Martínez-Contreras M. A comer... Diabetes insulino-dependiente. Bayer Health Care; 1996. Disponible en http://www.diabetes.bayer. es/user/default_alimentacion.htm

15. Brand-Miller J, Hayne S, Petocz P, Colagiuri $S$. Low-glycemic index diets in the management of diabetes. A meta-analysis of randomized controlled trials. Diabetes Care. 2003;26:2261-7.

16. Clarke $W$, Jones $T$, Rewers $A$, Dunger $D$, Klingensmith GJ. Assessment and management of hypoglycemia in children and adolescents with diabetes. Pediatric Diabetes. 2008;9:165-74.

17. Barrio, R. Actualización en el tratamiento de la diabetes mellitus tipo 1. An Pediatr Contin. 2003;1(1):21-6.

18. Golden MP, Ingersoll GM, Brack CJ, Russell BA, Wright JC, Huberty TJ. Longitudinal relationship of asymptomatic hypoglycemia to cognitive function in IDDM. Diabetes Care. 1989;12: 89-93.

19. Rovet JF, Ehrlich RM, Czuchta D. Intellectual characteristics of diabetic children at diagnosis 
and one year later. J Pediatr Psychol. 1990;15: $775-88$.

20. The diabetes control and complications trial research group. Effect of intensive diabetes treatment on the development and progression of long-term complications in adolescents with insulin-dependent diabetes mellitus: diabetes control and complications trial. Diabetes Control and Complications Trial Research Group. J Pediatr. 1994;125:177-88.

21. The diabetes control and complications trial research group. The relationship of glycemic exposure $(\mathrm{HbA} 1 \mathrm{c})$ to the risk of development and progression of retinopathy in the diabetes control and complications trial. Diabetes. 1995;44:96883.

22. Monnier L, Colette C. Glycemic variability: should we and can we prevent it? Diabetes Care. 2008;31 Suppl 2:s150-s154.

23. Svoren BM, Volkening LK, Butler DA, Moreland EC, Anderson BJ, Laffel LM. Temporal trends in the treatment of pediatric type 1 diabetes and impact on acute outcomes. J Pediatr. 2007;150: 279-85.

24. American Diabetes Association. Standards of medical care in diabetes. Diabetes Care. 2009;32: s13-s61.

25. Comisión de diabetes de la sociedad de endocrinología pediátrica de la AEP. Lo que debes saber sobre la diabetes infantil. Madrid: Ministerio de Sanidad y Consumo. Secretaría General Técnica; 2000. Disponible en http://www.fundaciondiabe tes.org/div/infantil/diab_infantil.htm

26. Laffel LM, Wentzell K, Loughlin C, TovaR A, Moltz K, Brink S. Sick day management using blood 3-hydroxybutyrate (3-OHB) compared with urine ketone monitoring reduces hospital visits in young people with T1DM: a randomized clinical trial. Diabet Med. 2006;23: 278-84.
27. Rewers $A$, Chase HP, Mackenzie $T$, et al. Predictors of acute complications in children with type 1 diabetes. JAMA. 2002;287:2511-8.

28. Rewers $M$, Pihoker $C$, Donaghue $K$, Hanas $R$, Swift P, Klingensmith GJ. Assessment and monitoring of glycemic control in children and adolescents with diabetes. Pediatric Diabetes. 2007;8: 408-18.

29. Brink S, Laffel L, Likitmaskul S, Liu L, Maguire AM, Olsen B, Silink M, Hanas R. Sick day management in children and adolescents with diabetes. Pediatric Diabetes. 2007;8:401-7.

30. Danne T, Aman J, Schober E, et al. A comparison of post and pre administration of insulin aspart in children and adolescents with type 1 diabetes. Diabetes Care. 2003;26:2359-64.

31. Brink, SJ. Diabetic ketoacidosis: prevention, treatment and complications in children and adolescents. Diabetes Nutr Metab. 1999;12:122-35.

32. Riddell M, Perkins B. Type 1 diabetes and vigorous exercise: applications of exercise physiology to patient management. Can J Diab. 2006;30 63-71.

33. Ligtenberg PC, Blans M, Hoekstra JB, Van der Tweel I, Erkelens DW. No effect of long-term physical activity on the glycemic control in type 1 diabetes patients: a cross-sectional study. Neth J Med. 1999;55:59-63.

34. Ebeling P, Tuominen JA, BoureY R, Koranyl $L$, KoivistO VA. Athletes with IDDM exhibit impaired metabolic control and increased lipid utilization with no increase in insulin sensitivity. Diabetes. 1995;44:471-7.

35. Tuominen JA, Karonen SL, Melamies L, Bolli G, Koivisto VA. Exercise-induced hypoglycaemia in IDDM patients treated with a short-acting insulin analogue. Diabetología. 1995;38: 106-11.

36. Admon G, Weinstein Y, Falk B, et al. Exercise with and without an insulin pump among chil- 
dren and adolescents with type 1 diabetes mellitus. Pediatrics. 2005;116:e348-55.

37. Delamater AM. Psychological care of children and adolescents with diabetes. Pediatric Diabetes. 2007;8:340-8.

38. Monge, MI. Psicología y diabetes. En: XIII Jornada de Diabetes del Niño y Adolescente de la SEEP VII Curso de Postgrado de Diabetes de la SEEP. 2008.
39. Kyngas $\mathrm{H}$, Hentinen $M$, Barlow $\mathrm{JH}$. Adolescents' perceptions of physicians, nurses, parents and friends: help or hindrance in compliance with diabetes self-care. J Adv Nurs. 1998;27:760-9.

40. Amillategui B, Calle JR, Álvarez MA, Cardiel $M A$, Barrio R. Identifying the special needs of children with Type 1 diabetes in the school setting. An overview of parents' perceptions. Diabet Med. 2007;24(10):1073-9. 